\title{
CAI-enhanced exam performance in a research design course
}

\author{
NANCY C. DUNCAN \\ Hampton University, Hampton, Virginia
}

\begin{abstract}
The efficacy of computer-assisted instruction (CAI) for increasing test performance in an experimental design course was tested using two commercially available programs to supplement course material. CAI was used to supplement course content for one of three examinations. Comparisons were also made with performance on the exams from 2 previous years. The results indicate a significant enhancement of performance by CAI.
\end{abstract}

A number of logical, insightful articles have been written on how computers could, theoretically, be effectively used to teach advanced courses in psychology (e.g., Butler, 1988; Castellan, 1986, 1988; Eamon, 1986; Peterson, 1989). However, only limited research on the efficacy of computer-assisted instruction (CAI) has been published, and the research which has been conducted has yielded mixed results. Some (Belmore, 1983; Goolkasian, 1989; Lowman, 1990; Ware \& Chastain, 1989) have found no effect of CAI on performance criteria when compared with that obtained with other methods of teaching. Other investigations have shown an enhanced effect of CAI on performance (Petty \& Rosen, 1990, 1991), on attitudes toward the course supported by CAI (Goolkasian, 1989; Ware \& Chastain, 1989), and on attitudes toward computers (Spivey, 1983). Welsh and Null (1991) found that traditional methods of teaching a laboratory component in a research design course were superior to computerbased methods of teaching.

Although a meta analysis on the effectiveness of CAI at the college level found moderate effects (Kulik \& $\mathrm{Ku}$ lik, 1987), the statement by Eamon (1986) that "there is precious little research showing that computer assisted instruction in psychology laboratory courses makes any difference at all," constitutes a valid summary of the present situation.

Given the expense and time required to implement CAI, it is imperative that more applied research be conducted to determine its efficacy. The research presented here describes the use and effectiveness of CAI in a laboratory course in research design.

\section{METHOD}

\section{Subjects}

The subjects were 139 junior and senior psychology majors enrolled in an advanced course in research design.

This research was partially supported by Grant P120A80053 from the Minority Science Improvement Program of the Department of Education. The author wishes to thank Perry Duncan for his editorial comments and Benton Gardner for assistance in data entry. Requests for reprints should be sent to Nancy C. Duncan, Department of Psychology, Hampton University, Hampton, VA 23668.

\section{Software and Apparatus}

Two software packages written by Fazio and Backler $(1986,1987)$ and available from CONDUIT, Power and Main Effects and Interactions, were used to teach concepts of research design. Power, a simulation based on an actual experiment, provides for the manipulation of six design features (increasing sample size, matching, use of aggregate measures, homogeneity of the sample, including an important source of variation as an independent variable and use of a pretest) to demonstrate how the power of an experiment is affected by the specific nature of its design. In Part 1, the student can compare the results (both $F$ and $p$ values are given) of changing each of these design variables with the outcome of a standard experiment. Part 2 allows students to design their own experiments. The number of possible outcomes is increased by the option given the student to either compare the results of their experiment with those of the standard one or to design two different experiments and compare the results.

Main Effects and Interactions is a tutorial on factorial designs, which contains an introduction to the concepts of main and interaction effects as well as practice exercises for determining whether such effects are significant. Data from one of the two social psychology experiments used as examples are displayed in either tables or graphs, which provides practice with reading both types of data display forms. Both two $(2 \times 2)$ and three-factor $(2 \times 2 \times 2)$ designs are included.

Software was available on 16 MS-DOS machines in the Psychology Department computer laboratory.

\section{Procedure}

Examination performance was evaluated over a 3-year period in which there was no CAI used in Year 1, limited $\mathrm{CAI}$ in Year 2, and more comprehensive CAI (and accompanying exercises) in Year 3 . In an attempt to control for extraneous variables, the same textbook, reading assignments, and lectures were used for all 3 years.

Scores on exams were used as the main performance criterion. All exams in the course contained a mixture of objective questions (30-55 items) and essay questions (2-3) in an approximate point composition of $70 \%$ to $80 \%$ 
objective and $30 \%$ to $20 \%$ essay. The multiple choice questions focused on specific facts, whereas essay questions required integration of information from several chapters. A typical essay question would require the student to design an experiment, to determine how to manipulate the IV, measure the DV, increase power, and/or to identify any potential ethical problems or limits to internal or external validity.

The exams for each of the 3 years contained identical multiple-choice questions and were carefully controlled during administration so as to maintain security. Since the essay questions were given to the students as a study aid, new ones were formulated each year.

Three exams were given in each course prior to the comprehensive final. Different information was covered in each exam in the course. CAI was used to supplement traditional teaching of the concepts covered on the second exam but not on the first and third exams. During Year 3, student complaints that Exams 1 and 2 were too long for the 50-min class period persuaded the instructor to shorten Exam 3 by $30 \%$.

Student lab assistants familiar with the software and the course were available to provide assistance for any student who had difficulty with any aspect of the assignment. They were instructed, however, not to provide answers for those who appeared unwilling to do the assignment. A handout describing the assignment for the Power software was given to each student.

There were some differences between Years 2 and 3 that should be noted. The computer lab was established during Year 2 and was not fully operational until the middle of the semester in which the present course was taught, making it necessary to quickly complete the CAI component. The lab assistants had thoroughly reviewed the software but had not actually used it as part of a course, and the instructor had had only limited experience using CAI.

During Year 3, two different types of assignments were used with the software packages because of the separate characteristics of each package. Power was accompanied by an assignment that required each student to run a number of simulations to determine the relative power of a variable or set of variables. The assignment required each student to find his or her own solution to the questions which had many possible correct answers. For example, one of the questions required the comparison of design features and the determination of the least effective procedure for increasing power. The appropriate answer depended on both the variables and the sequence in which they were manipulated. Grade determination was made on the basis of the written answers, which had to include a description of the strategies used and the supporting data.

The assignment for Main Effects and Interactions was less structured because of its format as a tutorial. Students were instructed to use the program to help themselves prepare for the upcoming exam. Credit was given to those who came to the lab and used the program. No time requirements were imposed.
Each year, a course evaluation tailored to the content and assignments of this particular course was administered to as many students as possible. A 7-point scale was used to assess course components such as lectures, use of CAI, computer facilities, exams, and so on, in which $1=$ very poor, $4=$ adequate, and $7=$ excellent . Information from Year 2 was used to improve the clarity and content of the Power assignment for use the following year. With a request that they be completed anonymously, the evaluations for Year 3 were mailed to the 40 students for whom summer addresses could be obtained.

\section{RESULTS}

Mean exam scores for each of the 3 years are presented in Table 1.

A two-way analysis of variance (ANOVA) with repeated measures yielded highly significant effects for year $[F(2,136)=18.57, p<.0001]$, exams $[F(2,272)=$ $31.04, p<.0001]$, and the interaction $[F(4,272)=6.74$, $p<.0001]$.

Individual ANOVAs for each year (Bruning \& Kintz, 1977) were run to determine if CAI increased learning as demonstrated by superior scores on Exam 2 for Years 2 and 3.

The results of the ANOVA for Year 1 were significant $[F(2,64)=4.90, p<.01]$. Individual comparisons using Scheffé's tests revealed a significant difference between Exams 1 and $2(p<.01)$ but not for Exams 2 and 3 or 1 and 3 .

A significant $F$ was also found for Year $2[F(2,102)=$ $5.41, p<.005]$. Planned comparisons showed a significant difference only between Exams 1 and $3(p<.006)$.

The results of the ANOVA for Year 3 were significant $[F(2,106)=45.66, p<.0001]$, with Scheffé's test yielding significant results for all comparisons (Exam 1 vs. Exam 2, $p<.0001$; Exam 2 vs. Exam 3, $p<.0001$; Exam 1 vs. Exam 3, $p<.0028$ ).

An analysis of test questions for Exam 2 showed that $23 \%$ of the objective questions and $45 \%$ of the essay questions, for a total of $33 \%$ of the entire test, were related to the CAI material.

Twenty-three of the evaluations for Year 3 were returned, for a return rate of $57 \%$. Sixty-three percent of the students receiving a B or an A returned the evaluations; only $20 \%$ of those making $\mathrm{C}$ or below did so. Results of the student evaluations for Year 3 indicated that

Table 1

Descriptive Statistics for Exams 1-3 for Years 1-3

\begin{tabular}{ccccccccc}
\hline & \multicolumn{2}{c}{ Year 1(1988) } & & \multicolumn{2}{c}{ Year 2(1989) } & & \multicolumn{2}{c}{ Year 3 (1990) } \\
\cline { 2 - 3 } \cline { 7 - 8 } Exam & $M$ & $S E M$ & & $M$ & $S E M$ & & $M$ & $S E M$ \\
\hline 1 & 59.53 & 2.27 & & 66.51 & 1.82 & & 50.81 & 1.60 \\
2 & 67.03 & 1.81 & & 70.06 & 1.36 & & 66.17 & 1.82 \\
3 & 62.91 & 2.32 & & 72.74 & 1.71 & & 56.54 & 1.55 \\
$n$ & \multicolumn{2}{c}{33} & & \multicolumn{2}{c}{52} & & \multicolumn{2}{c}{54} \\
\hline
\end{tabular}


satisfaction with the computer component (mean $=5.56$ ) was similar to that for other assignments (research proposal $=5.68$, research project $=5.26$, and review of journal articles $=5.43$ ). When asked to identify parts of the course that were particularly helpful, $61 \%$ of the students listed the computer exercises, $48 \%$ the research project, and $9 \%$ the review of journal articles. In response to a question regarding components in need of improvement, only $9 \%(n=2)$ indicated the computer exercises.

\section{DISCUSSION}

The results for Year 3 show that performance on the second exam, in which the content of text and lectures was supported by CAI, was significantly superior to that of both Exam 1 and Exam 3.

The significant difference between Exams 2 and 3 is particularly compelling for two reasons. First, the format of all exams was similar, and any practice effect would clearly be in favor of Exam 3 . Second, Exam 3 was $30 \%$ shorter than the first two. Student feedback at a midsemester course evaluation revealed almost unanimous agreement that the exams were too long. Exam 3 was consequently shortened from $\mathbf{4 5}$ multiple-choice items to 30 and from 3 essay questions to 2 . This change in exam length was made in the interests of effective and fair teaching over confound-free experimental design. Student performance on Exam 3 was indeed superior to that on Exam 1, as indicated by the results of the Scheffé test, but not to that on Exam 2 .

As Butler (1988) and Castellan (1988) have stated, it is essential to select software that is appropriate for a particular course. Toward this end, software that supports course objectives, including teaching appropriate content, is more likely to be effective. The software used in this course was selected to support three objectives: (1) to reinforce concepts discussed in text and lectures, (2) to provide an opportunity for exploration of the relationship between experimental design and consequent results, and (3) to provide practice in interpreting data sets presented in different forms.

The performance of the students on the assignment based on the Power software was impressive. The majority of the papers $(76 \%)$ were of B quality or better, with $40 \%$ receiving a perfect score. These results provide evidence that objectives 2 and 3 were met. Inspection of the papers revealed many different approaches in fulfillment of the assignment, indicating that each student was exerting individual effort. Thus, the results of this experiment provide evidence that the CAI component was effective in meeting all three objectives.

It is possible, however, that one of several uncontrolled variables might have produced the results we obtained. Clark (1983), in a review critical of much research on the effectiveness of educational media, argues that rival hypotheses usually exist, hypotheses such as differences in teaching methods, content, and the novelty of the medium currently in vogue. The present research attempted to control for some of these confounding variables by using the same instructor, who endeavored to maintain constant teaching technique and content throughout the study. Additionally, the results show that material covered on the second exam was not inherently easier to learn, since performance on it was not significantly superior during the first 2 years of this study.

If novelty were an important factor, it seems that the biggest effect should have occurred in Year 2, when the computers were first used to support courses in the Psychology Department. Students taking the course in Year 3 were familiar with CAI, having encountered it in two previous courses in psychology. Only after refinement of the CAI assignment and its sequence in the course did a significant performance effect occur.

Students taking the course during Year 3 of the study unexpectedly had lower scores on Exams 1 and 3 than did those in Years 1 and 2. CAI may be a more effective aid to learning for students experiencing difficulty in a course.

The programs used in this experiment are "older generation" software which have been available for some time. The zeitgeist in recent software development has favored the creation of complex, often difficult-to-learn experiment simulators (for example, MEL and MacLab) with great power and flexibility but unknown efficacy for teaching research design. It is hoped that the results of the present study will encourage those considering the development of innovative and effective data generators or tutorials and will stimulate well-controlled investigation of the efficacy of the various types of CAI.

\section{REFERENCES}

Belmore, S. E. (1983). Release from PI: Comparison of traditional and computer modules in an experimental psychology laboratory. Behavior Research Methods, Instruments, \& Computers, 15, 191-194.

BruniNG, J. L., \& KINTZ, B. L. (1977). Computational handbook of statistics (2nd ed). Glenview, IL: Scott, Foresman.

BUTLER, D. L. (1988). Selection of software in the instructional laboratory. Behavior Research Methods, Instruments, \& Computers, 20, 175-177.

Castellan, N. J. (1986). Issues in the effective use of computers in introductory and advanced courses in psychology. Behavior Research Methods, Instruments, \& Computers, 18, 251-256.

Castellan, N. J. (1988). Comments on applications of microcomputers in teaching. Behavior Research Methods, Instruments, \& Computers, 20, 193-196.

Clark, R. E. (1983). Reconsidering research on learning from media Review of Educational Research, 53, 445-459.

EAMON, D. B. (1986). Integrating a computer component into the student psychology laboratory: Problems and prospects. Behavior Research Methods, Instruments, \& Computers, 18, 245-250.

FAzio, R. H., \& BACKLER, M. H. (1986). Topics in research methods: Main effects and interactions. Iowa City, IA: University of Iowa, Conduit.

FAzIO, R. H., \& BACKLER, M. H. (1987). Topics in research methods: Power. lowa City, IA: University of Iowa, Conduit.

Goolkasian, P. (1989). Computerized laboratories for psychology instruction: How successful are they? Behavior Research Methods, Instruments, \& Computers, 21, 148-150. 
Kulik, J. A., KulIK, C.-L. C. (1987). Review of recent research literature on computer-based instruction. Contemporary Educational Psychology, 12, 222-230.

LowMAN, J. (1990). Failure of laboratory evaluation of CAI to generalize to classroom settings: The SuperShrink interview simulation. Behavior Research Methods, Instruments, \& Computers, 22, 429-432.

Peterson, S. B. (1989). A microcomputer lab for psychology based on a hierarchical learning model. Behavior Research Methods, Instruments, \& Computers, 21, 130-133.

Petty, L. C., Rosen, E. F. (1990). Increase in mastery levels using a computer-based tutorial/simulation in experimental psychology. Behavior Research Methods, Instruments, \& Computers, 22, 216-218.
Petty, L. C., \& Rosen, E. F. (1991). Using HyperCard to teach person perception and attribution concepts. Behavior Research Methods, Instruments, \& Computers, 23, 247-248.

SPIVEY, J. E. (1983). Software development for computer-assisted instruction in experimental psychology. Behavior Research Methods, Instruments, \& Computers, 15, 186-196.

Ware, M. E., * Chastain, J. D. (1989). Computer-assisted statistical analysis: A teaching innovation? Teaching of Psychology, 16. 222-227.

WeLSH, J. A., NULL, C. H. (1991). The effects of computer-based instruction on college students' comprehension of classic research. Behavior Research Methads. Instruments, \& Computers, 23, 301-305.

\title{
Notices and Announcements
}

\author{
Submission of Manuscripts on Computer Disk
}

To facilitate publication, the Publications Office of the Psychonomic Society strongly urges authors to provide floppy disks with the final, accepted paper (hard copy) versions of their manuscripts. The texts in the two should, of course, agree exactly.

Please note that ALL DISK FILES SHOULD BE IN ASCII FORMAT (ASCII is referred to by some word processing software as "nondocument" or "text-only" mode). Also, supply all manuscript materials in one file.

Please indicate the following on the disk label:

1. File name, and suffix (if any).

2. Type of disk and actual formatted capacity:

DOS $51 \frac{1}{4}$ in.: $180 \mathrm{~K}$ (SS, DD) or $360 \mathrm{~K}$ (DS, DD)

DOS $51 / 4$ in.: $1.2 \mathrm{M}$ (DS, HD)

DOS $31 \frac{1}{2}$ in.: $360 \mathrm{~K}$ (SS, DD or $720 \mathrm{~K}$ (DS, DD)

DOS $31 \frac{12}{2}$ in.: $1.44 \mathrm{M}$ (DS, HD)

Macintosh (400K or $800 \mathrm{~K}$ only; we cannot use high-density Macintosh disks)

3. Word processing software used and version number. (Note that we cannot use MacWrite.)

4. Editor's manuscript number, our production number, or first author's surname.

Please also note the following: (1) Each Heading, Paragraph, Figure Caption, and Reference should be followed by two (2) "hard" returns, including the hard return marking the end of a heading, paragraph, etc. (i.e., press the "enter" or "return" key twice). (2) Delete all running heads, page numbers, indications of figure/table placements, etc., from the disk. (3) If you use predefined functions, such as the $F($ unction)5 key, in lieu of a formatting command string, please be as consistent as possible in their use. Converting files to ASCII does not always remove all of these embedded commands, but those repeated consistently are more easily removed in typesetting. (4) Use the numeral for the number 1 (one), and not a lowercase 1 (el).

Finally, before you send your disk, please verify that your file has been copied correctly. 